\title{
The Conundrum of 'Long-COVID-I9': A Narrative Review
}

Mandeep Garg, (D' Muniraju Maralakunte, (iD)' Suruchi Garg, (iD) ${ }^{2}$ Sahajal Dhooria, ${ }^{3}$ Inderpaul Sehgal, ${ }^{3}$ Ashu Seith Bhalla, ${ }^{4}$ Rajesh Vijayvergiya, ${ }^{5}$ Sandeep Grover, ${ }^{6}$ Vikas Bhatia,' Priya Jagia, ${ }^{7}$ Ashish Bhalla, ${ }^{8}$ Vikas Suri, ${ }^{8}$ Manoj Goyal, ${ }^{9}$ Ritesh Agarwal, ${ }^{3}$ Goverdhan Dutt Puri, ${ }^{10}$ Manavjit Singh Sandhu'

'Department of Radiodiagnosis \& Imaging, PGIMER, Chandigarh, India; ${ }^{2}$ Department of Dermatology, Aura Skin Institute, Chandigarh, India; ${ }^{3}$ Department of Pulmonary Medicine, PGIMER, Chandigarh, India; ${ }^{4}$ Department of Radiodiagnosis, AllMS, New Delhi, India; ${ }^{5}$ Department of Cardiology, PGIMER, Chandigarh, India; ${ }^{6}$ Department of Psychiatry, PGIMER, Chandigarh, India; ${ }^{7}$ Department of Cardiovascular Imaging \& Endovascular Interventions, AlIMS, New Delhi, India; ${ }^{8}$ Department of Internal Medicine, PGIMER, Chandigarh, India; 'Department of Neurology, PGIMER, Chandigarh, India;

${ }^{10}$ Department of Anaesthesia and Intensive Care, PGIMER, Chandigarh, India

\section{Video abstract}

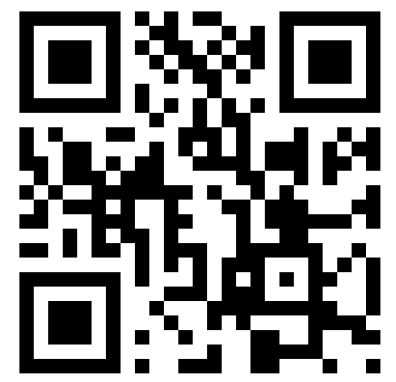

Point your SmartPhone at the code above. If you have a $Q R$ code reader the video abstract will appear. Or use: https://youtu.be/rSFIP8bHkXO

Correspondence: Mandeep Garg Department of Radiodiagnosis and Imaging, PGIMER, Sector-12, Chandigarh, India Tel +91 9914209390

Email gargmandeep@hotmail.com
Abstract: COVID-19 is an ongoing pandemic with many challenges that are now extending to its intriguing long-term sequel. 'Long-COVID-19' is a term given to the lingering or protracted illness that patients of COVID-19 continue to experience even in their post-recovery phase. It is also being called 'post-acute COVID-19', 'ongoing symptomatic COVID-19', 'chronic COVID-19', 'post COVID-19 syndrome', and 'long-haul COVID-19'. Fatigue, dyspnea, cough, headache, brain fog, anosmia, and dysgeusia are common symptoms seen in Long-COVID-19, but more varied and debilitating injuries involving pulmonary, cardiovascular, cutaneous, musculoskeletal and neuropsychiatric systems are also being reported. With the data on Long-COVID-19 still emerging, the present review aims to highlight its epidemiology, protean clinical manifestations, risk predictors, and management strategies. With the re-emergence of new waves of SARS-CoV-2 infection, LongCOVID-19 is expected to produce another public health crisis on the heels of current pandemic. Thus, it becomes imperative to emphasize this condition and disseminate its awareness to medical professionals, patients, the public, and policymakers alike to prepare and augment health care facilities for continued surveillance of these patients. Further research comprising cataloging of symptoms, longer-ranging observational studies, and clinical trials are necessary to evaluate longterm consequences of COVID-19, and it warrants setting-up of dedicated, post-COVID care, multidisciplinary clinics, and rehabilitation centers.

Keywords: ongoing symptomatic COVID-19, post-COVID-19 syndrome, chronic COVID, Long-COVID-19, post-COVID, Long-COVID

\section{Introduction}

Corona Virus Disease 2019 (COVID-19), caused by severe acute respiratory syndrome coronavirus 2 (SARS-CoV-2), is emerging out to be a pandemic full of mysteries, where understanding its pathogenesis is one task; and as the time is passing by, the post COVID19 complications being unveiled are the next level challenge. ${ }^{1,2}$ According to the World Health Organization (WHO), almost $80-85 \%$ of COVID-19 infections are mild or asymptomatic and resolve completely like any other uncomplicated seasonal flu, 10$15 \%$ are severe illness requiring hospitalization/oxygen supplementation, and about 5\% are critical illness requiring intensive care and mechanical ventilation. ${ }^{3}$ Generally, the majority of the patients show complete recovery within 3-4 weeks of COVID-19 infection, but few patients continue to experience its lingering effects and develop protracted illness/medical complications that can have lasting health problems.

It is called 'Long-COVID-19' - a term first used by a patient Elisa Perego from Italy, as a hashtag on Twitter in May 2020 while describing her own experience with continuing symptoms of COVID-19 even after recovery. However, it has moved through various social media to formal clinical experts and policymakers and has gained reasonable 
consistency. Recent joint guidelines proposed by the National Institute for Health and Care Excellence (NICE), the Scottish Intercollegiate Guidelines Network (SIGN), and the Royal College of General Practitioners (RCGP) have divided COVID-19 infection into 3 phases - 'Acute COVID-19' (signs and symptoms of COVID-19 infection up to 4 weeks), 'ongoing symptomatic COVID-19' (from 4 weeks up to 12 weeks), and 'post-COVID-19 syndrome' (when signs and symptoms continue beyond 12 weeks). ${ }^{4}$ The term 'LongCOVID-19' is given to the signs and symptoms that continue or develop after the 'acute COVID-19' phase and include both 'ongoing symptomatic COVID-19' and 'post COVID-19 syndrome'. The 'chronic COVID' and 'long-haul COVID' are the other names used to describe the sequel of COVID$19 .^{5-7}$ But, these terms are limited only to describe the clinical stages of recovery and are unrelated to the underlying active illness or infectivity. It is also unclear whether these represent a new syndrome specific to COVID-19 or any overlap with post-viral syndrome or post-intensive care syndrome (PICS) in some patients.

It is currently estimated that approximately 3.3 million people worldwide have succumbed to COVID-19 while 140 million people have "recovered", 8 but whether these patients have "truly recovered" remains evasive. Fatigue, dyspnea, cough, anosmia, brain fog, and dysgeusia are the most common symptoms encountered in Long-COVID-19, but organ-system-specific injuries involving pulmonary, cardiovascular, cutaneous, and neuropsychiatric system have also been reported. However, the limited data on patient followup make it difficult to precisely measure the long-term debilitating health and economic consequences of COVID-19. There is no wide awareness of the Long-COVID-19 case scenarios even among the healthcare workers. Thus, it becomes imperative to emphasize this emerging clinical entity and disseminate its awareness to the medical professionals, patients, public, and policymakers alike; and prepare and augment the healthcare facilities to address the footprints marked by this disease in recovered subjects.

An extensive review of the available literature was done using different databases, including Google Scholar, PubMed, Web of science, bioRxiv, medRxiv, and ResearchGate, references from relevant articles, and internet sources (WHO reports) till 25th April 2021. Search terms included LongCOVID-19, post-COVID, chronic COVID, post-COVID syndrome and long-haul COVID, viral illness following COVID19, post-COVID illness, COVID recovery, predictors of LongCOVID-19. A total of 212 articles out of 5846 screened manuscripts were utilized for this comprehensive review. Most of the studies evaluated specific symptoms based on previously applied questionnaire/surveys in patients recovered from COVID-19 and included admixture of both hospitalized and non-hospitalized patients of mild, moderate and severe COVID-19. Based on the current scientific evidence, the present article comprehensively reviews the epidemiology, etiopathogenesis, clinical manifestations, predictors, and management strategies in COVID-19 survivors in their convalescent/recovery phase. We have also shared the clinical images of patients with Long-COVID-19 from our personal experience with COVID-19 in the two apex, tertiary care medical institutes of India having fully dedicated COVID-19 hospital wings. Informed written consent from the patients was obtained.

\section{Epidemiology of Long-COVID-I 9}

There is lack of systematic reporting of the long-term consequences of COVID-19 with data still emerging and availability of only few early studies on the subject. The nascent data from the subjects with COVID-19 related cardiac and neurological ailments; and the past epidemic/pandemic experience with respiratory illnesses due to other viral infections hint at the potential persistence of the symptoms with delayed return to the baseline health status. In a recent systematic review and meta-analysis, 80\% (CI 65-92) patients with a confirmed COVID-19 diagnosis are reported to continue having at least one symptom beyond two weeks following acute infection. ${ }^{9}$

A pilot study from India reported an average time for COVID-19 recovery as 25 days after onset of disease in adult subjects, and there was no significant difference noted between gender and across different age groups for recovery time. ${ }^{10}$ Two recent prospective studies reported persistence of symptoms in 32\% of the outpatient COVID-19 subjects from Swiss $(n=669)$, and $83 \%$ of hospitalized COVID-19 subjects from Italy $(n=143) .{ }^{11,12}$ The office for National Statistics, the UKbased survey, reported the prevalence of Long-COVID-19 symptoms of 1 in 5 COVID positive subjects for five weeks or longer, and 1 in 10 COVID positive subjects for 12 weeks or longer. $^{13}$ Fatigue (11.5\%), cough (11.4\%), and headache $(10.1 \%)$ are the common symptoms reported in the survey. Additionally, it also revealed the increased incidence of metabolic and medical (cardiovascular, renal, and hepatic conditions) diseases in hospitalized COVID-19 subjects compared with demographic and clinical profile matched other subjects. $^{13}$

Interestingly, Long-COVID-19 symptoms are infrequently seen in children as compared to adults aged more than 40 years. There was no statistically significant association found among 
the different adult age groups/race of the subjects with the manifestations of Long-COVID-19. ${ }^{12,14-19}$ The early followup studies up to 3 months of acute illness revealed dyspnea and fatigue as major symptom profiles; while a longer follow-up study at seven months reported fatigue (78\%), post-exertional malaise (72\%), and cognitive dysfunction (55\%) as most frequent Long-COVID-19 symptoms in that order. ${ }^{12,14-19}$ Davis et al also reported the compromised quality of life in people at seven months following acute illness; and reported that $45.2 \%$ of subjects required reduced workload compared to their preillness state, while $22.3 \%$ of the subjects had still not returned for work. ${ }^{19}$ However, the majority of these survey-based studies done to evaluate the demographic and clinical features in the follow-up cohorts of the Long-COVID-19 subjects have reported major participation from the females, western people, and those with high education and higher socioeconomic class.

Lopez-Leon et al reported 55 long-term effects associated with COVID-19 in their recent systematic review and metaanalysis of 21 studies consisting of 47,910 patients of LongCOVID-19. ${ }^{9}$ They found that fatigue (58\%), headache (44\%), attention disorder (27\%), hair loss (25\%) and dyspnea (24\%) were the 5 most common persistent symptoms in patients recovering from COVID-19 in decreasing order of frequency. ${ }^{9}$ There was female predilection for fatigue, postactivity polypnea, and alopecia symptoms. However, no other symptoms revealed any gender association in this meta-

Table I Summary of Demographic and Clinical Features from the Follow-Up Studies of Long-COVID-19 Subjects

\begin{tabular}{|c|c|c|c|c|c|c|c|}
\hline Study Characteristics & $\begin{array}{l}\text { Carfi } \\
\text { A et al. }\end{array}$ & $\begin{array}{l}\text { Goërtz YMJ } \\
\text { et al. }{ }^{14}\end{array}$ & $\begin{array}{l}\text { Garrigues } \\
\text { E et al. }{ }^{15}\end{array}$ & $\begin{array}{l}\text { Xiong } \\
\text { et al. }{ }^{16}\end{array}$ & $\begin{array}{l}\text { Dennis } \\
\text { et al. }{ }^{17}\end{array}$ & $\begin{array}{l}\text { Carvalho- } \\
\text { Schneider et al. }{ }^{18}\end{array}$ & $\begin{array}{l}\text { Davis HE } \\
\text { et } \text { al }^{19}\end{array}$ \\
\hline Sample size (number) & 143 & 2113 & 120 & 538 & 201 & 130 & 3,762 \\
\hline Age (Yrs.) & 56.5 & 47 & 63.2 & NR & 44 & NR & NR \\
\hline Gender (M:F) (number) & 90: 53 & $310: 1803$ & $75: 45$ & 245: 293 & $61: 140$ & 82: 48 & $718: 296 \mid$ \\
\hline $\begin{array}{l}\text { Co-morbidities (number } \\
\text { of ailments) }\end{array}$ & 124 & 820 & 82 & 177 & 100 & 44 & $N R$ \\
\hline Follow-up period (Days) & $\begin{array}{l}60 \text { (After } \\
\text { onset) }\end{array}$ & $\begin{array}{l}79 \text { (After } \\
\text { onset) }\end{array}$ & $\begin{array}{l}\text { II } 0.9 \text { (After } \\
\text { admission) }\end{array}$ & $\begin{array}{l}90 \text { (After } \\
\text { discharge) }\end{array}$ & $\begin{array}{l}\text { I40 (After } \\
\text { onset) }\end{array}$ & 60 (After onset) & $\begin{array}{l}>180 \text { (After } \\
\text { onset) }\end{array}$ \\
\hline \multicolumn{8}{|l|}{ Persisted symptoms } \\
\hline Dyspnea & $43.4 \%$ & $89.5 \%$ & $41.7 \%$ & NR & $87.1 \%$ & $7.7 \%$ & $37.9 \%$ \\
\hline Fatigue & $53.1 \%$ & $94.9 \%$ & $55 \%$ & $28.3 \%$ & $98 \%$ & NR & $77.7 \%$ \\
\hline Anosmia & NR & $39.7 \%$ & $13.3 \%$ & NR & $\mathrm{N} / \mathrm{A}$ & $22.7 \%$ & \multirow{2}{*}{$\begin{array}{l}25.2 \% \\
\text { (Combined) }\end{array}$} \\
\hline Dysgeusia & NR & $42.3 \%$ & $10.8 \%$ & NR & $\mathrm{N} / \mathrm{A}$ & $22.7 \%$ & \\
\hline Chest pain & $21.7 \%$ & $N / A$ & $10.8 \%$ & $12.3 \%$ & $73.1 \%$ & $13.1 \%$ & $32.9 \%$ \\
\hline Cough & NR & $68.1 \%$ & $16.7 \%$ & $7.1 \%$ & $73.6 \%$ & NR & $20.1 \%$ \\
\hline $\begin{array}{l}\text { Cognitive impairment/ } \\
\text { Brain fog }\end{array}$ & $N R$ & $N / A$ & $61 \%$ & $N R$ & NR & NR & NR \\
\hline Anxiety & NR & NR & NR & $6.5 \%$ & NR & NR & NR \\
\hline Joint pain & $27.3 \%$ & $38.2 \%$ & $N R$ & $7.6 \%$ & $78.1 \%$ & $16.3 \%$ & NR \\
\hline Hair loss & NR & NR & $20 \%$ & $28.6 \%$ & NR & NR & NR \\
\hline Skin problems & $N R$ & NR & $N R$ & NR & NR & $11.5 \%$ & NR \\
\hline Diarrhea & NR & $41.1 \%$ & $N R$ & NR & $59.2 \%$ & $33.3 \%$ & $N R$ \\
\hline Palpitation & NR & $54.9 \%$ & $N R$ & $11.2 \%$ & $N R$ & $N R$ & $40.1 \%$ \\
\hline Sleep disorders & NR & NR & $30.8 \%$ & NR & NR & NR & NR \\
\hline
\end{tabular}

Abbreviation: NR, not reported. 
analysis. ${ }^{9}$ Table 1 enlists the demographic and clinical features of Long-COVID-19 reported in various studies.

\section{Etiopathogenesis of Long-COVID-I 9 with Newer Insights}

The understanding of the pathophysiology of Long-COVID -19 is important to predict, prevent and treat long-term consequences of COVID-19. The etiology of Long-COVID-19 seems to be a continuum of the disease pathogenesis of acute illness. To understand this continuity, it is important to first know how COVID-19 affects the various organ systems in its acute phase. SARS-CoV-2 has a special affinity for angiotensin-converting enzyme-2 (ACE-2) receptors found in abundance in organs such as lungs, liver, kidneys, and blood vessels. The virus primarily affects the respiratory system, and after reaching the lung alveoli, it attaches and penetrates the host cell, particularly type II pneumocytes, and starts damaging the alveoli. It erodes into the capillary endothelium, causing endotheliitis and leads to the formation of microthrombi; ${ }^{1,20}$ then enters the blood vessels and may disseminate in the whole body. The virus also stimulates the inflammatory milieu in the body, causing significant release of cytokines and chemokines leading to cytokine storm in severe cases. A few authors have opined about association of mast cell activation syndrome and resultant cytokine storm with Long-COVID-19. ${ }^{21}$ The resultant organ damage caused by such excessive inflammatory response takes much longer to recover and is responsible for the symptoms of LongCOVID-19. ${ }^{20}$ The underlying vasculitic patho-mechanism is also supported by observations in a prospective study with FDG-PET/CT reporting higher target-to-blood pool ratio at aorto-iliac arterial tree in Long-COVID-19 subjects as compared to the control group. ${ }^{22}$

There is a possibility that the virus may be entering into vessels in the nose or throat itself in its earliest phase, considering anosmia and dysgeusia are experienced in the initial phase of the illness, and injury to the olfactory bulb has even been demonstrated on MRI. ${ }^{1,23,24}$ In some individuals, these injuries may take much longer to heal resulting in continuation of its symptoms even in post-recovery phase. Another important mechanism of extrapulmonary manifestations in COVID-19 is the formation of antigen-antibody complexes by the second or third week when the humoral immunity gets activated. ${ }^{25}$ The role of autoimmunity secondary to viral infections is a well-known phenomenon and organ damage in COVID-19 appears to be largely immune-mediated, similar to other autoimmune diseases. ${ }^{26-29}$ Garg et al postulated occurrence of antigen-antibody reactions in COVID-19 and predicted it to be occlusive and propagative injuries; ${ }^{1}$ which also explains why some patients continue to suffer from varied and debilitating symptoms for many months after infection.

A study from China on COVID-19 patients admitted in ICU documented anti-52 kDa Ro/SSA (Sjögren's-syndromerelated antigen A), anti-60 kDa Ro/SSA, and ANA (antinuclear antibodies) in $20 \%, 25 \%$, and $50 \%$ of patients, respectively. ${ }^{30}$ These autoantibodies aggravate the ongoing insult in pulmonary tissues. They can be responsible for pulmonary fibrosis, and other long-term post-COVID consequences occurring in a few patients. ${ }^{31}$ US-based health researchers have reported the immune dysregulation behind the prothrombotic state and its related conditions in COVID-19 subjects. ${ }^{32,33}$ Though the exact etiology of Long-COVID-19 is still unclear, it appears secondary to endotheliopathy, hypoxemic injuries, antigen-antibody reactions, or aberrant immune response.

Reactivation or reinfection of the SARS CoV-2 is another concern in the follow-up period, with few such cases being reported lately. ${ }^{34,35}$ While its possible mechanisms are still under investigation; one explanation could be that neutralizing antibodies in individuals recovered from SARS-CoV-2 infection start waning within 2-3 months after infection. ${ }^{36}$ This observation suggests that some of the Long-COVID-19 subjects can still be the carriers of the virus and may require an additional round of virus detection, thereby necessitating the need for close monitoring and follow-up of such patients. Serial antibody determination and evidence of active viral replication might be used to provide additional support and to further characterize SARS-CoV-2 reinfections. ${ }^{35}$

\section{Manifestations of Long-COVID- 19}

With the COVID-19 pandemic raging across the world, many recovered patients continue to suffer the lingering effects of the infection, including respiratory, cardiac, cutaneous, and nervous system impairment. Persistence of the physical symptoms seen in acute viral illness is the most common manifestation of Long-COVID-19. Sometimes, a flare-up of the neuropsychiatric symptoms or appearance of new psychiatric symptoms is reported in $16-18 \%$ patients of Long-COVID-19, especially those who had critical illness or ICU admission. ${ }^{37}$ LongCOVID-19 subjects with persistent symptoms have reported poor quality of life and impaired functionality in $40 \%$ of the hospitalized patients even 60 days following discharge. ${ }^{38}$ The common clinical manifestations encountered in Long-COVID-19 are illustrated in Figure 1. 


\section{Long-COVID-19 : Common Manifestations}

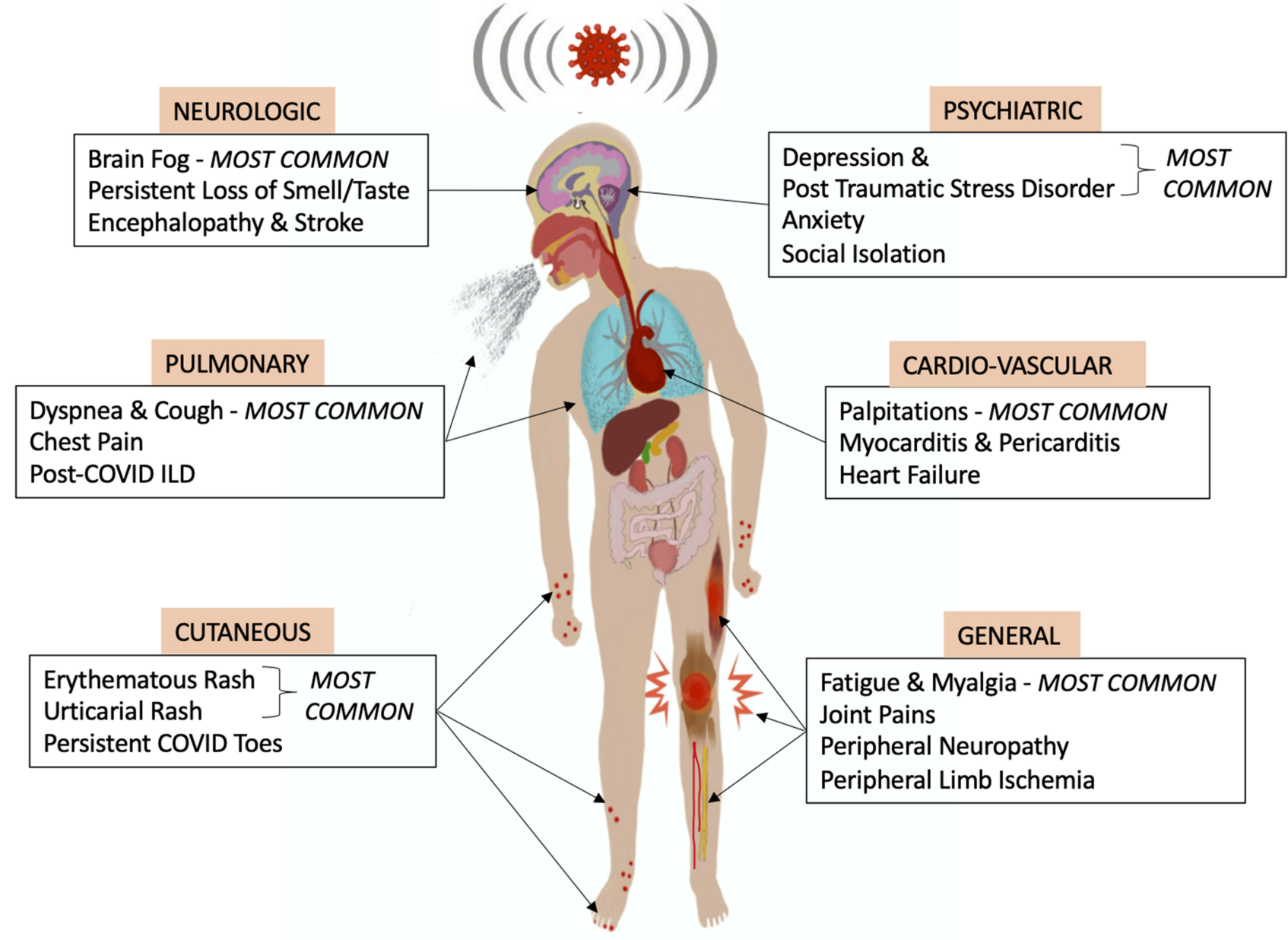

Figure I A pictorial illustration of the common clinical manifestations observed in Long-COVID-I9.

\section{General Manifestations}

These are non-specific but often experienced persistent symptoms of COVID-19, even by patients with a milder form of the disease. Persistence of some of the acute illness physical symptoms, including dyspnea, fatigue, post-exertional malaise, chest pain, and cough, are the most common manifestation of the Long-COVID-19. ${ }^{12,14-19}$ An onlinequestionnaire-based study on 2113 post-COVID-19 patients reported only $0.7 \%$ of symptom-free subjects at 79 days after acute COVID-19 pneumonia. ${ }^{14}$ It also reported fatigue (87\%) and dyspnea ( $71 \%$ ) as the most prevalent symptoms at 79 days after onset of acute illness. ${ }^{14}$ A survey-based study consisting of post-discharged hospitalized subjects $(\mathrm{n}=143)$ found fatigue (53\%), dyspnea ( $43 \%)$, loss of memory (34\%), sleep disorders (30.8\%), impairment of concentration (28\%), and joint pain $(27 \%)$ as the frequently reported persistent symptoms at 110 days after discharge from the hospital. ${ }^{15}$ The other cardiopulmonary, musculoskeletal, gastrointestinal, and neuropsychiatric symptoms are reported in the multiple studies during the post-recovery phase between 60 to 180 days. ${ }^{16-19}$ Davis et al also revealed the probability of having prolonged symptoms of moderate, severe, and very severe category noted in $36.6 \%, 14.5 \%$, and $5.2 \%$ of the Long-COVID-19 patients six months after the onset of acute illness. Further, it also stated that fatigue, dyspnea, and cognitive abnormalities (brain fog) as the major debilitating manifestations leading to the decreased quality of life. ${ }^{19}$ A meta-analysis by Lopez-Leon also reported the derangement of laboratory parameters like D-dimer and N-terminal pro-brain natriuretic peptide (NTproBNP) in $20 \%$, C-reactive protein (CRP) in $11 \%$, serum ferritin in $8 \%$, procalcitonin in $4 \%$ and interleukin-6 (IL-6) in $3 \%$ of Long-COVID- 19 patients. ${ }^{9}$

\section{Pulmonary Manifestations}

The respiratory system is the most common organ-system affected by the SARS-CoV-2, and cough and dyspnea are 
the most frequent pulmonary complaints reported by the Long-COVID-19 subjects. However, the most worrisome health concern is the development of post-COVID-19 interstitial lung disease (PC-ILD) in few patients. These patients can present with persistent hypoxia and abnormal pulmonary function tests. CT chest is an invaluable diagnostic tool in these patients which can show a sequel of organizing pneumonia or features of lung fibrosis manifesting as persistent ground-glass opacities (GGOs) and reticulations, traction bronchiectasis/bronchiolectasis, and rarely honeycombing ${ }^{39}$ (Figures 2 and 3). A meta-analysis by Garg et al has shown GGOs and consolidation as the most common CT findings in acute COVID-19, the persistence of which has been reported by several authors at the time of discharge/during follow-up. ${ }^{40,41}$ The direct viral injury to the respiratory epithelia, complicated by the cytokine storm and pulmonary prothrombotic events, leads to lung injury and its sequel following an acute viral illness. Also, hyperbaric oxygen therapy, ventilatorinduced injury, hospital-acquired infection and superadded, or reactivation of the systemic infections are the significant factors associated with the persistence or emergence of the new pulmonary manifestations in follow-up period. $^{42}$ In a cohort of 61 patients, Chun et al showed that immuno-fibrotic markers such as lipocalin-2, hepatocyte growth factor and matrix metalloproteinase-7 were higher in ICU patients and showed inverse relationship with the lung function tests. ${ }^{43}$

Wang et al reported the clearance of lung abnormalities on CT scan only in 4/70 subjects at the time of discharge, and the remaining 66/70 subjects showed residual findings. Persistent ground-glass abnormalities were reported in $60 \%$ of patients. ${ }^{3941}$ Mo et al reported pulmonary function assessment on 110 discharged subjects. The abnormal diffusion capacity of the lung for carbon monoxide (DLCO) was seen in $47.2 \%$ of patients and reduced total lung capacity (TLC) in $25 \%$ of the subjects. The DLCO reduction was in a linear relationship with the increasing severity of the illness. ${ }^{44}$ In another study, the pulmonary function assessment revealed abnormal results in 14 (25.4\%) subjects, with impaired DLCO, FVC, FEV1, and TLC in $9(16.3 \%), 6(10.9), 6(10.9)$, and $4(7.2 \%)$ of the recovered subjects, respectively. ${ }^{45}$

\section{Cardiac Manifestations}

It is a well-recognized fact that COVID-19 affects the cardiovascular system, and post-recovery can lead to myocarditis (Figure 4), acute/decompensated heart failure, acute coronary syndrome, arrhythmias, and

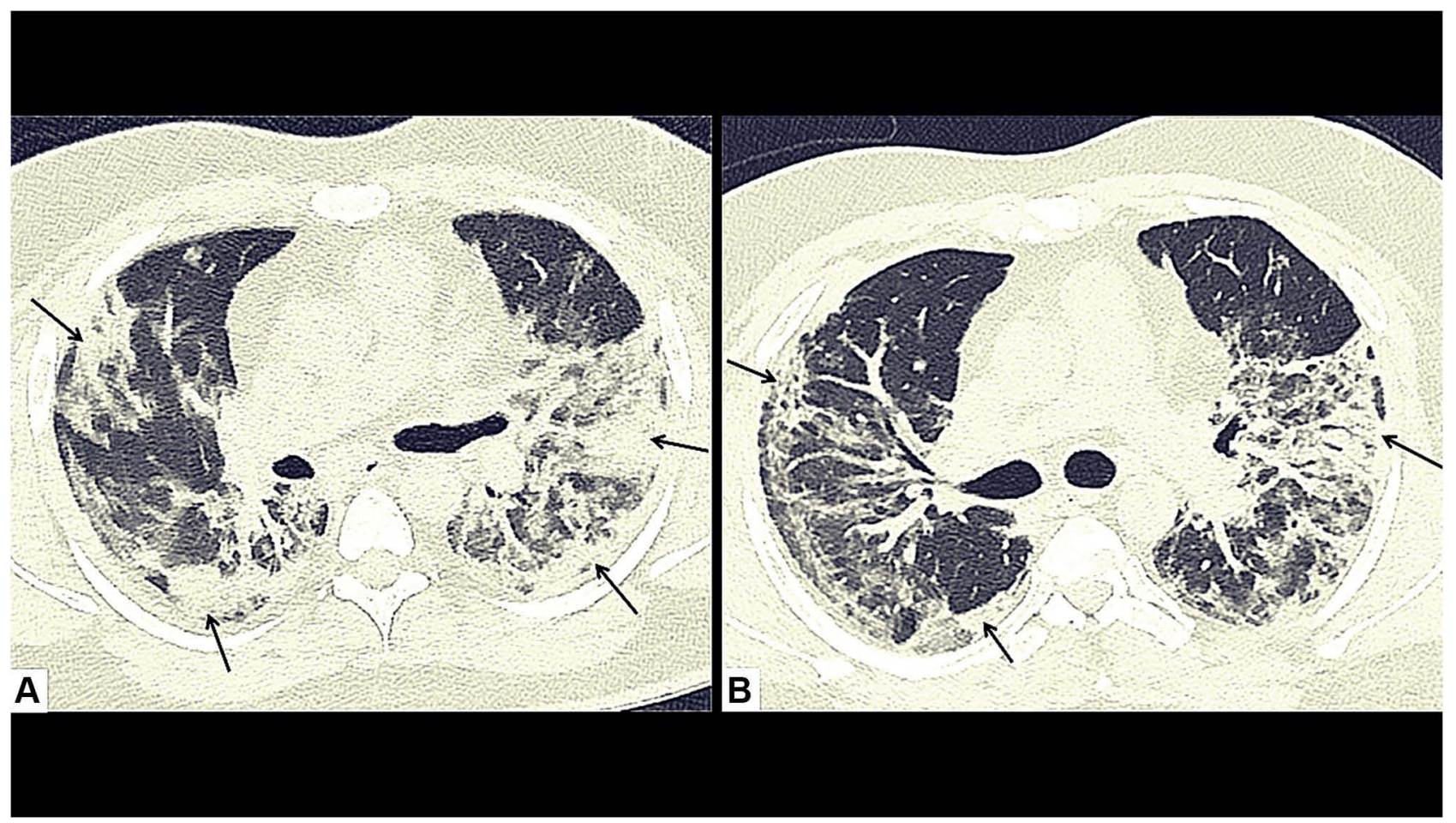

Figure 2 A 45-year-old male, a reformed smoker, and occasional alcohol consumer with sequel of COVID-19 pneumonia. The baseline CT chest at the time of admission, axial image (A) showing peripheral areas of consolidation (black arrows) with surrounding ground glass opacities (GGOs) in bilateral upper lobes. He had persistent cough even at the time of discharge, and follow-up CT chest (6 weeks after discharge), axial image (B) showing resolution in peripheral consolidation, but persistent area of interstitial thickening (black arrows) in bilateral lungs. 


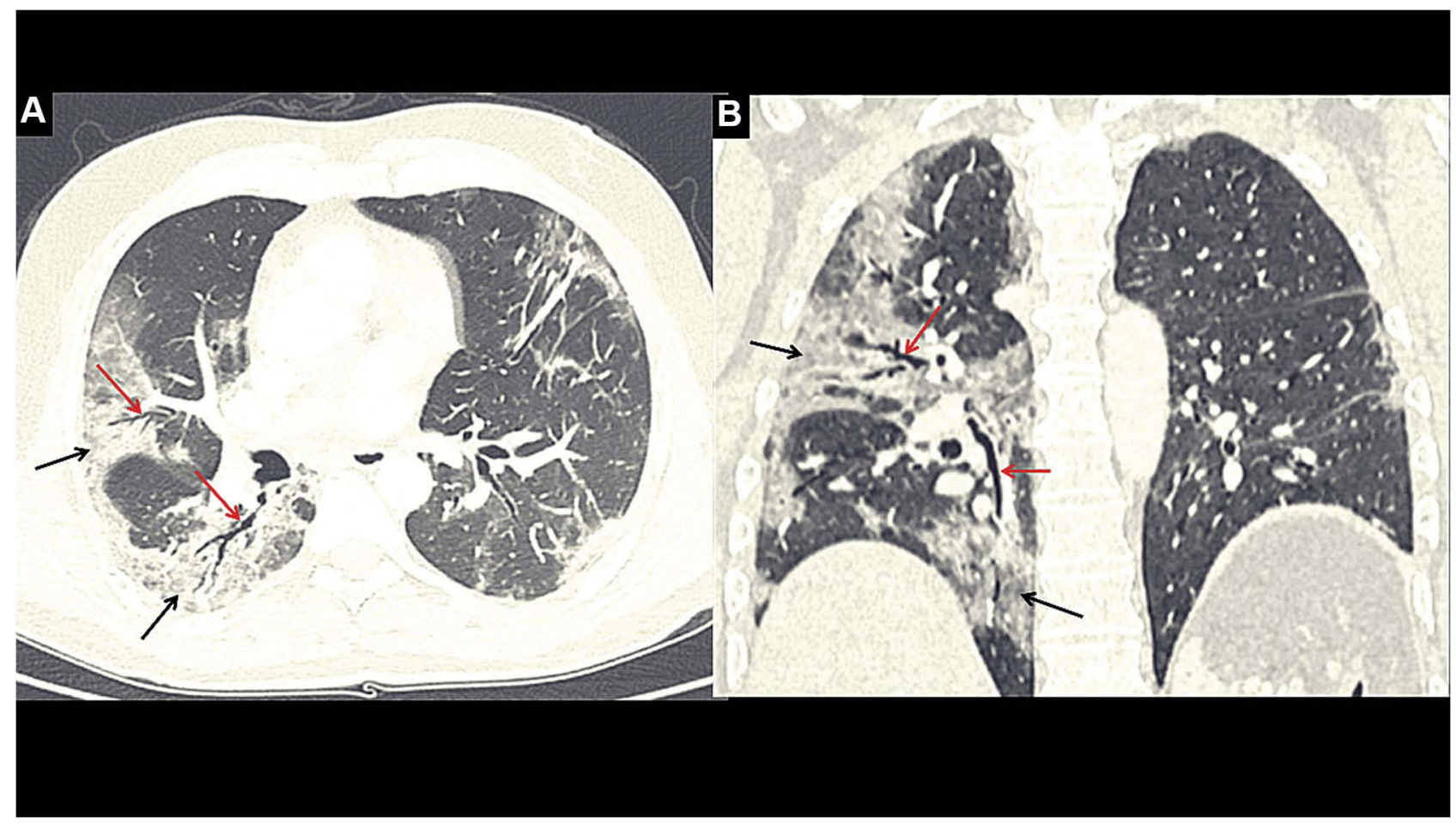

Figure 3 A 48-year-old male who was hospitalized for COVID-19 pneumonia, presents with persistent dyspnea and fatigue after 8 weeks of discharge. High resolution computed tomography (HRCT) chest, axial (A) and coronal (B) reformatted images, showing bilateral peripheral areas of ground glass opacity (GGO) (black arrows) and interstitial thickening (right $>$ left) with traction bronchiectasis (red arrows) in right middle and lower lobe.

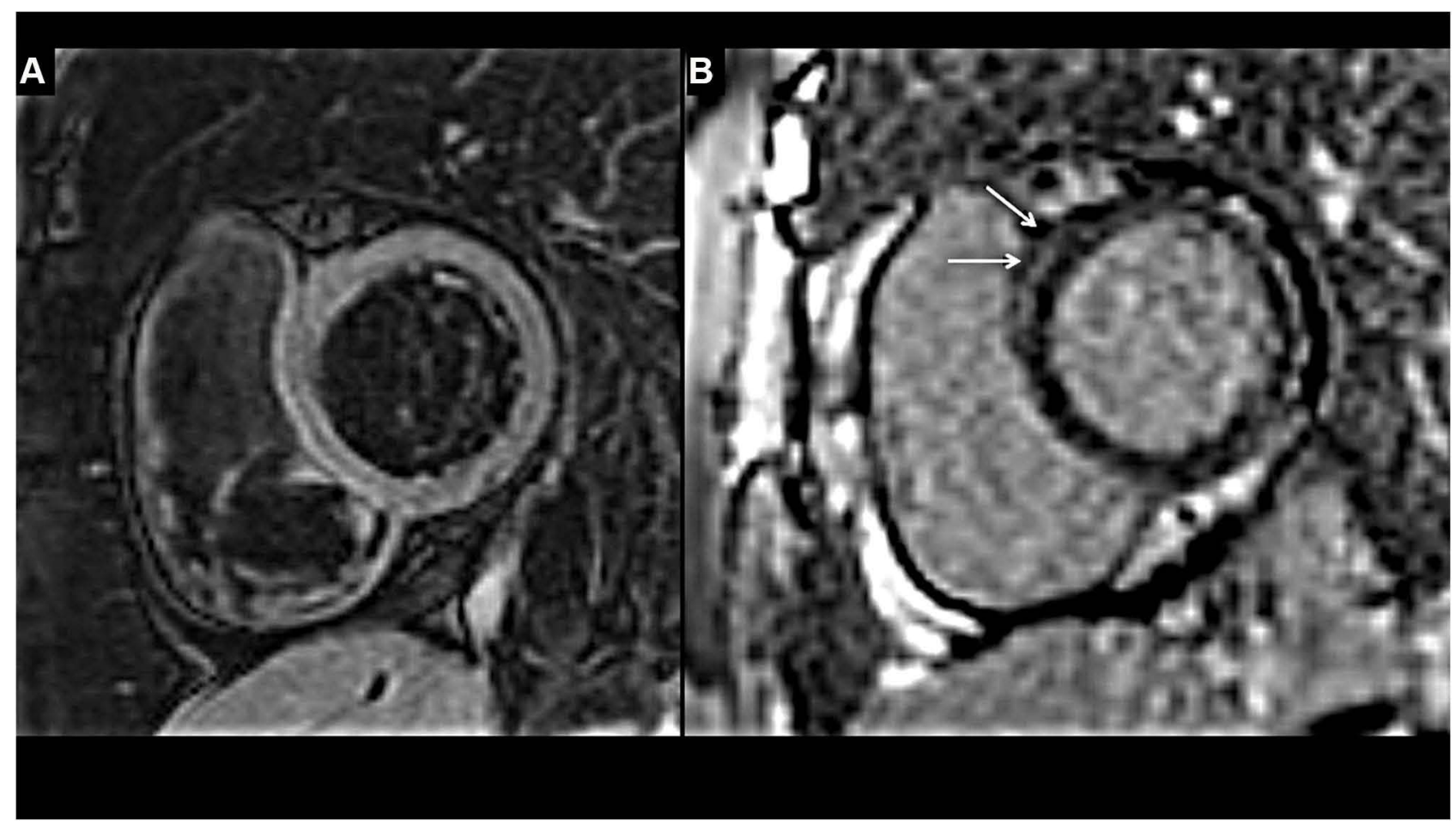

Figure 4 Cardiac MRI images in a 45-year-old female patient with Long-COVID-19 myocarditis, at 5 weeks after discharge from hospital: (A) fat saturated T2- weighted image in short axis view at basal left ventricle (LV) shows normal myocardial signal intensity, suggesting absence of edema. (B) Late gadolinium enhancement (LGE) PSIR sequence in short axis view done at 15 minutes post contrast shows presence of linear LGE in anteroseptal segment of the basal LV (white arrows), denoting presence of fibrosis, likely sequel of myocardial injury due to the prior COVID infection. 
thromboembolic events in subjects with and without preexisting cardiovascular disease. ${ }^{46}$ Sudden cardiac arrest has also been reported in few patients recovered from COVID-19. The predominant underlying mechanism of cardiac injury in acute COVID-19 is mainly by an indirect advanced systemic inflammation, endotheliitis, and type-II myocardial infarction due to hypoxia. ${ }^{47-49}$ The same ongoing smouldering inflammation is thought to be responsible for the resultant cardiac manifestations of Long-COVID-19.

Huang L and colleagues reported the abnormal cardiac MRI findings in the cohort of recovered, moderate to severely ill COVID-19 subjects $(n=26)$, who had initial cardiac symptoms. ${ }^{50}$ Cardiac MR was done at a median interval of 47 days from the onset of cardiac symptoms. Findings included abnormal signal changes on STIR sequence and late gadolinium enhancement (LGE) suggestive of edema and myocardial fibrosis/scarring, respectively; seen in $58 \%$ of the subjects. The basal and mid, inferior, and inferolateral segments were the common sites of LGE reported in the study. ${ }^{50}$

Another prospective study conducted by the German radiologists on the Long-COVID-19 cohort $(n=100)$ included patients recovered from home (67\%) as well as hospitalization (33\%) with a median duration of 71 days after COVID-19 diagnosis. ${ }^{51}$ It revealed abnormal cardiac $\mathrm{MR}$ in $78 \%$ of subjects, including reduced left ventricular ejection fraction, LGE, pericardial enhancement, and raised myocardial native $\mathrm{T} 1$ and $\mathrm{T} 2$ values. The raised myocardial native $T 1$ value $(n=73)$, raised myocardial native T2 value $(n=60)$, myocardial LGE $(n=32)$, or pericardial enhancement $(n=22)$ findings are noted in decreasing order of frequency. ${ }^{51}$ Another cardiac MR study, done on COVID-19 recovered, competitive athletes $(n=26)$, revealed myocarditis (high T2 value and LGE) and patchy LGE - as an isolated finding (fibrosis) in 15\% and $30.8 \%$ of the subjects, respectively, with a total $45.8 \%$ of subjects with abnormality. ${ }^{52}$ Autonomic dysfunction causing resting tachycardia in Long-COVID-19 has also been described by a case series. ${ }^{53}$ The exact pathogenesis is unknown currently, and authors recommended prompt recognition and supportive care for long haulers with autonomic dysfunction. $^{53}$

\section{Cutaneous Manifestations}

The skin is an open window to look into the body's internal environment, and cutaneous manifestations can give an important clue to the sequel of COVID-19. An international registry for COVID-19 dermatological manifestations has reported various COVID-19 related skin manifestations including morbilliform rashes, urticarial eruptions, and papulo-squamous lesions. ${ }^{54}$ It has been found that urticarial and morbilliform rashes are short lasting, whereas the pernio $(6.8 \%)$ and livedo-reticularis lasted for more than 60 days. ${ }^{54}$ Altered microangiopathy of peripheral arterial system supplying hands, foot, and digits is believed to be associated with chilblain-like lesions (pernio) in Long-COVID-19; and a few authors have advocated for a research evaluating the nailfold capillaroscopy of COVID toes to understand the altered microvascular hemodynamics. ${ }^{55}$ The drug-related dermatological eruptions cannot be overlooked while evaluating Long-COVID-19 subjects, and sometimes, even the previously existent dermatosis can also get complicated ${ }^{56}$ (Figure 5).

\section{Neurological Manifestations}

The neurological manifestations are seen in half of the hospitalized patients, with a higher proportion of reported complications in critically ill subjects, often seen to persist in the post-recovery phase. These can vary from mild symptoms like headache, myalgia, weakness, vertigo, chemosensory dysfunction (anosmia/ageusia); to more serious complications like seizures, encephalopathy, and stroke. ${ }^{57}$ Brain fog is another common manifestation of LongCOVID-19, an umbrella term used to describe the constellation of cognitive function impairment such as confusion, short-term memory loss, dizziness, and inability to concentrate. ${ }^{19,59}$ It is thought to be secondary to the SARS$\mathrm{CoV}-2$ causing hypoxia and mitochondrial dysfunction that leads to microstructural brain damage and resultant symptoms of brain fog. ${ }^{58}$

A multicenter European study has revealed residual olfactory and gustatory dysfunction in 53.9 and $22.5 \%$ of the recovered subjects, respectively, and $23.6 \%$ of the subjects exhibited both olfactory and gustatory impairment. ${ }^{59}$ A recent study has revealed cerebral (involving the olfactory cortices, insulae, and hippocampi) microstructural changes in COVID-19 recovered subjects at three months of follow-up using the diffusion tensor imaging (DTI) technique. ${ }^{60}$ Encephalopathy, agitation and corticospinal tract signs are another persistent finding seen in Long-COVID-19, however, at present, it is difficult to ascertain whether these are secondary to COVID-19 related damage or just a sequel of critical illness. ${ }^{61}$ The other critical illness-related neurological manifestations such as hypoxic white matter injury and cerebral 


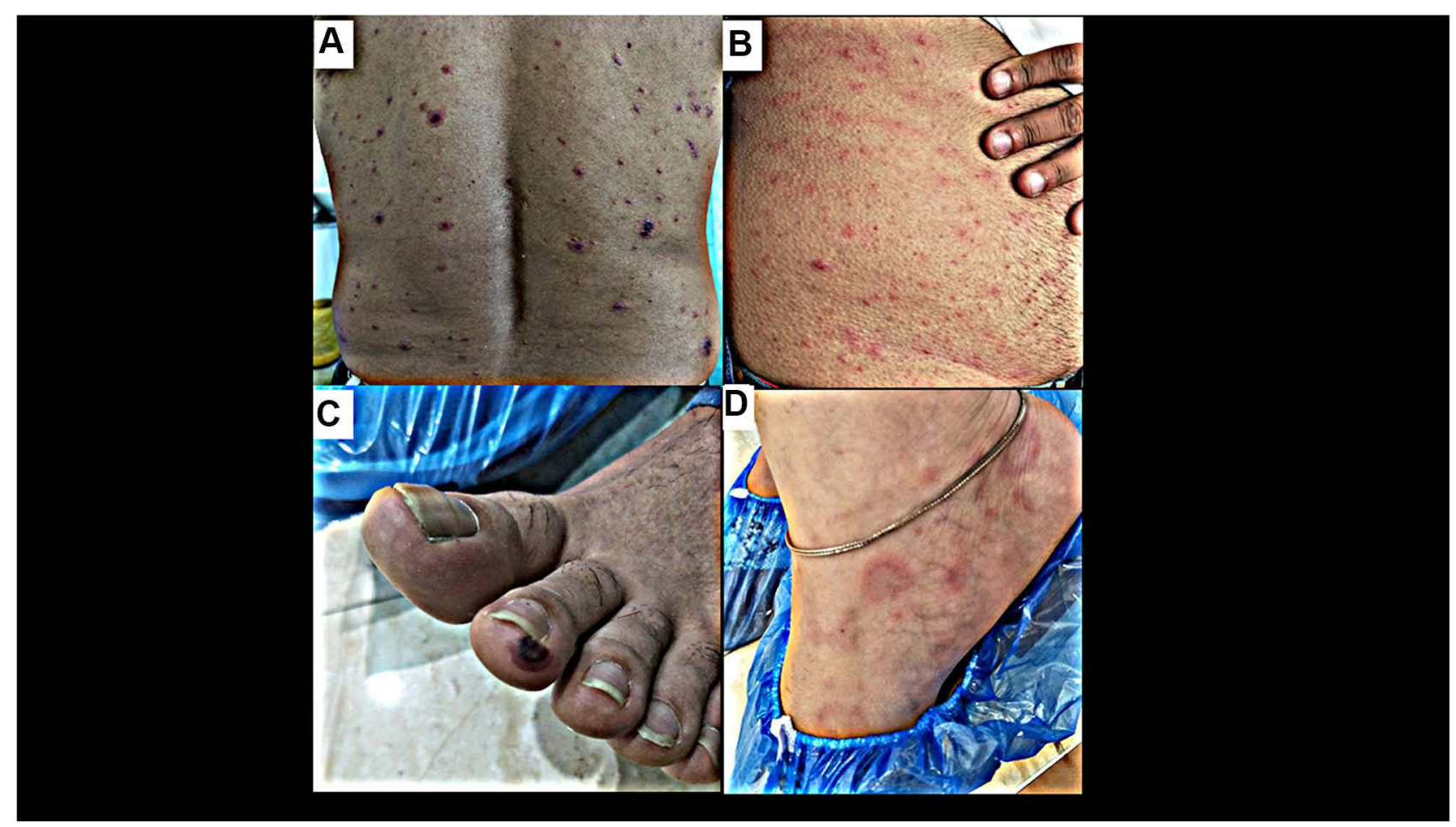

Figure 5 Cutaneous manifestations of Long-COVID-19 in different patients after varying duration of initial diagnosis: (A) hemorrhagic guttate psoriatic lesions on the back in previously known psoriasis patient (5 weeks after symptom onset) (B) retiform maculopapular itchy rash on the trunk (10 weeks after disease onset) (C) persistent, asymptomatic COVID toe (12 weeks after initial diagnosis) (D) erythema multiforme lesions on the right foot (6 weeks following symptom onset).
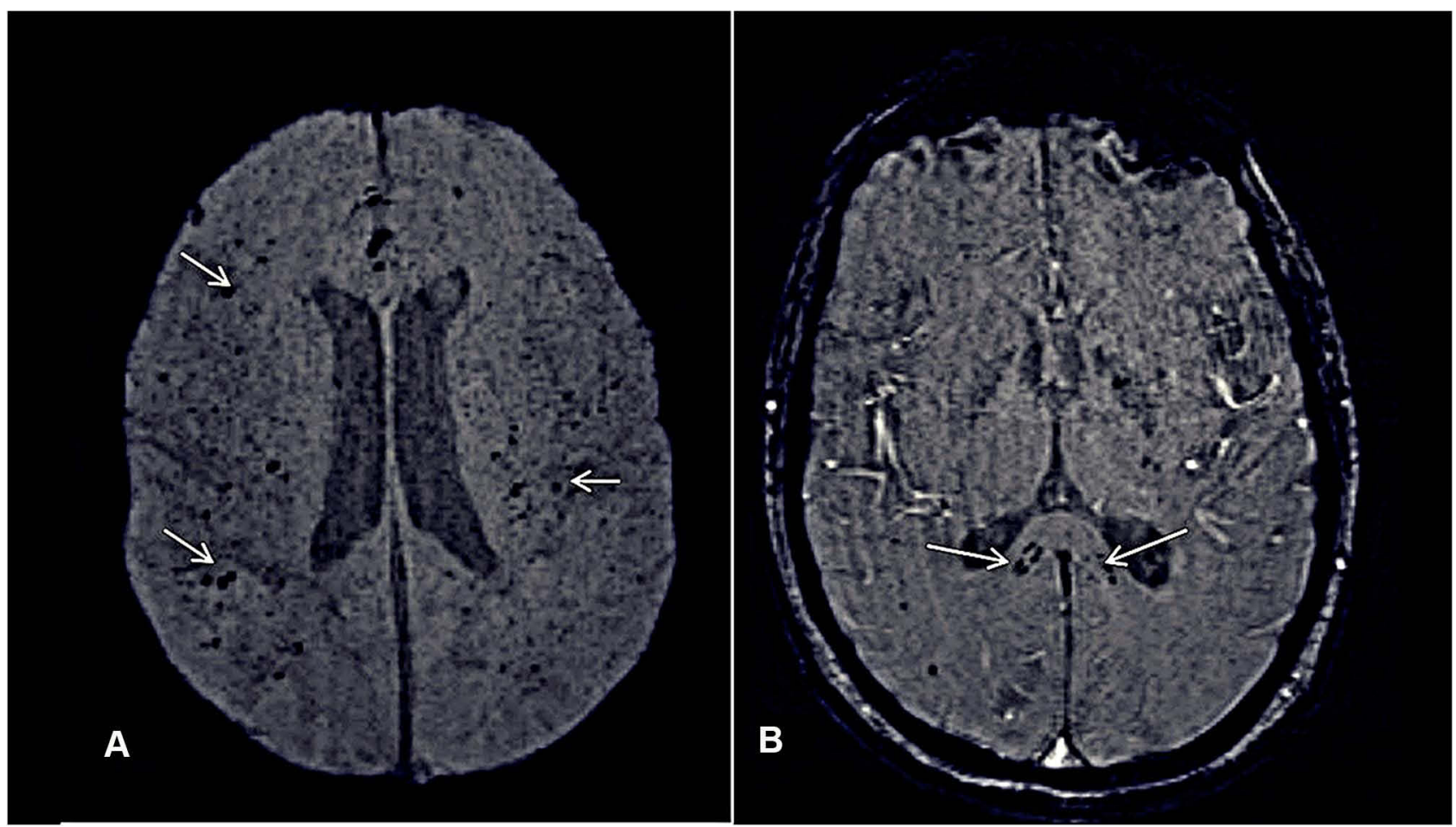

Figure 6 53-year male patient with history of COVID-19 induced ARDS and prolonged ICU stay. Follow-up (12 weeks after discharge) MRI brain for altered sensorium shows presence of multiple microbleeds on SWI images seen as multiple hypointense foci (white arrows) predominantly at grey-white matter junction (A) and corpus callosum (B) likely suggestive of critical illness induced microbleeds. 
microbleeds are also not uncommon in COVID-19 survivors (Figure 6).

\section{Neuropsychiatric Manifestations}

The impaired cognition, attention, concentration, and sleep are the commonly reported neuropsychiatric manifestations in the post-recovery phase of COVID-19. The causes of neuropsychiatric consequences of post-COVID-19 infection are multifactorial - effects of encephalitis/cerebral hypoxia, medical interventions, physical isolation, psycho-social impact, and social stigma, to name a few. ${ }^{62}$ The hyper-inflammatory status associated with the SARS CoV-2 infection (high levels of CRP, IL-6, and ferritin) is another important factor predisposing to psychiatric morbidities like depression. ${ }^{63}$ The true incidence/prevalence of the mental, emotional, and behavioral abnormalities in Long-COVID-19 subjects is yet to be explored. The neuropsychiatric literature on survivors of critical illness has unmasked the prevalence of post-traumatic stress disorder (PTSD), anxiety, and depressive symptoms in $34 \%, 34 \%$, and $29 \%$ of the subjects, respectively. ${ }^{64-66}$ Hopkins and colleagues reported impaired attention span, mental processing speed, concentration, and memory at one-year following the post-acute respiratory syndrome. ${ }^{67}$ In another study, one-third of the patients (15/45) with severe COVID-19 pneumonia showed dysexecutive syndrome, including the features of inattention, poorly organized motor tasks, and disorientation. ${ }^{61}$

\section{Miscellaneous Manifestations}

The endocrinological, prothrombotic, retinal, and neuromuscular complications are lately being reported in Long-COVID-19 subjects. Sansone et al have revealed the long-term concerns of male sexual and reproductive health (erectile dysfunction) in COVID-19 survivors. ${ }^{68}$ Though its exact cause is not yet elucidated, the impaired cardiovascular and pulmonary hemodynamics, endothelial dysfunction, psychological factors, and subclinical hypogonadism are the suspected reasons behind the onset of erectile dysfunction. ${ }^{68}$

A few recent studies have also reported acute limb ischemia, renal artery thrombosis, retinitis, and optic neuritis in COVID-19 subjects and its survivors. ${ }^{69-71}$ The reported neuromuscular complications like peripheral nerve injuries are predominantly idiopathic, though these can be secondary to the underlying systemic disease/ immune dysregulation, direct neural-invasion, or sometimes even related to the hospital-care (like prone positioning to treat ARDS). ${ }^{72}$ MRI and high-resolution sonographic evaluation of the affected peripheral nerve is suggested, guiding the appropriate treatment and rehabilitative therapy in such individuals. ${ }^{72}$

\section{Predictors of Long-COVID-I 9}

At this stage of the pandemic, the researchers are steering towards understanding the long tail of recovery and postrecovery phase of the COVID-19. The currently available data is sparse to analyze the predictors of Long-COVID-19 development, as the studies addressing the post-recovery phase are limited in number, with the majority of them being survey-based analysis. Various factors such as language, sociodemographic variations, and lack of awareness about the follow-up study apps can hinder factors from accurately analyzing the natural course of LongCOVID-19. The initial reports from China, with acute COVID-19 illness data, have described a high case fatality rate in hospitalized subjects with advanced age $(>70$ years), with a critical illness (severity), and in patients with underlying co-morbid conditions (including cardiovascular disease, diabetes, respiratory ailments, cancer). ${ }^{73}$ Smoking, chronic alcoholism, and patients with prolonged ICU stay/mechanical ventilation are the other factors reported to be associated with Long-COVID-19. ${ }^{74}$ Recent studies are unveiling the underlying altered immune mechanisms for development and progression of COVID-19 illness. Altered distribution of the ACE- 2 and CD 147 related genes are reported from subjects with asthma, obesity, chronic obstructive pulmonary disease (COPD), hypertension, smoking and male gender in relation to development of severe COVID-19 illness. ${ }^{75,76}$ More recently studies have made a striking revelation that even the non-hospitalized subjects with mild acute illness and without any significant co-morbidities are also experiencing prolonged symptoms and multi-organ derangement following acute COVID-19. ${ }^{17,77}$ The various risk predictors have been summarized in Figure 7.

\section{Age and Gender}

People of all ages are affected by the SARS-CoV-2 virus; however, several cohorts have reported that the hospitalization rate of COVID-19 increased with advancing age, with a rate of $4 \%$ for subjects of $50-59$-years versus $18 \%$ for those above 80 -years of age and these patients are at an increased risk of developing symptoms of Long-COVID $-19{ }^{78,79}$ A primitive study targeting the subjects with mild illness (non-hospitalized) revealed a significant proportion 


\section{Predictors / Risk Factors for Long-COVID}

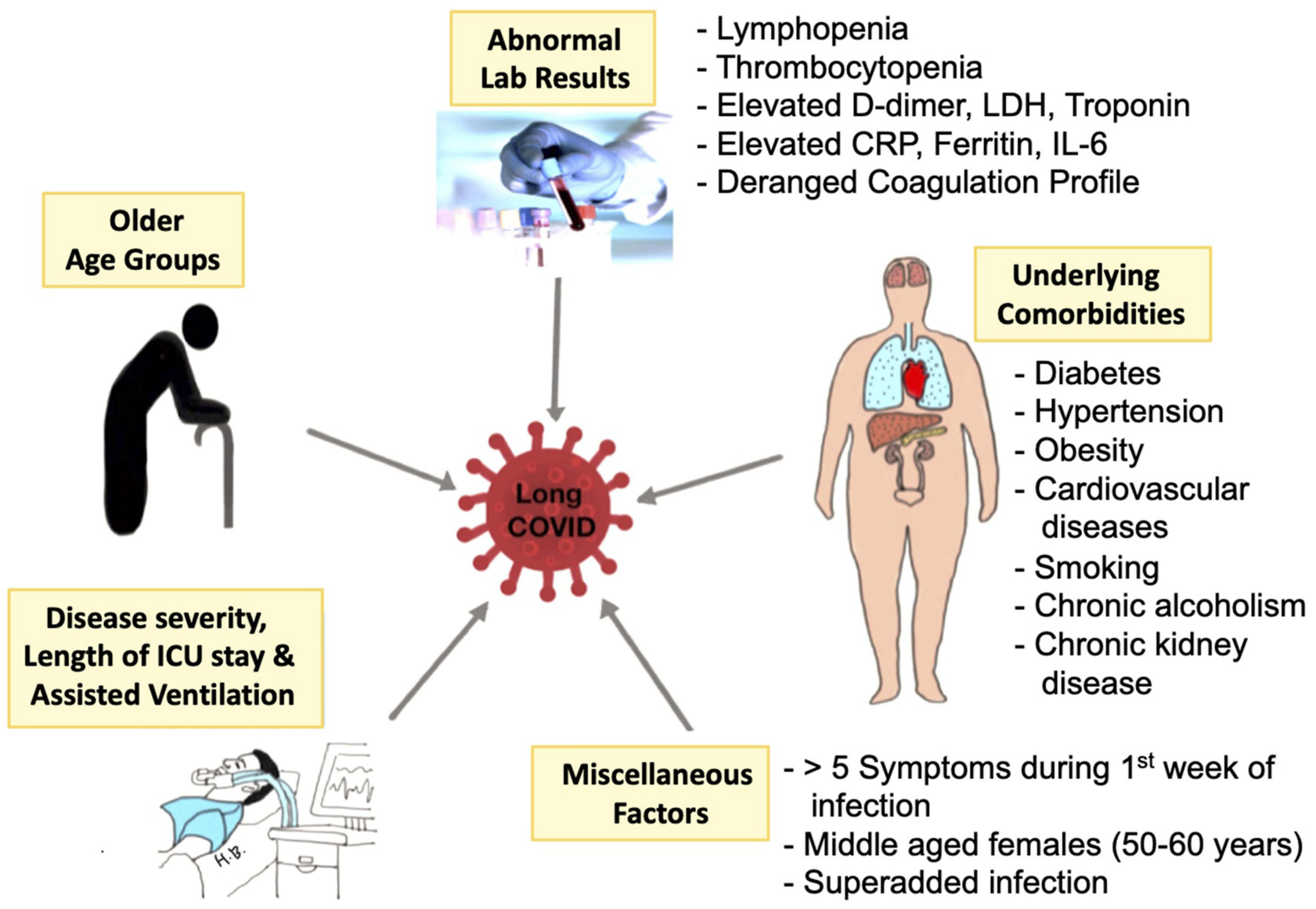

Figure 7 A pictorial demonstration of the various risk factors which when present, make the patients of COVID-19 more susceptible to develop Long-COVID-I9.

(47\%) of subjects with age above 50 years, not returning to their usual health than $26 \%$ of subjects between age 18-34 years. ${ }^{80}$ Another study, by Sudre et al reported a rise in the prevalence of Long-COVID-19 from $10 \%$ of subjects in the age group of $18-49$ years to $22 \%$ in those aged above 70 years. ${ }^{81}$ It also revealed that the slightly high proportion (15\%) of Long-COVID-19 in middle-aged females (50-60 years) as compared to the male population (10\%); however, such a gender-related variance is not observed in the subjects with age more than 70 years. ${ }^{81}$

\section{Underlying Co-Morbidities}

Based on the analysis of 300,000 COVID-19 subjects, a US study reported 12 times higher mortality among the subjects with co-morbid conditions compared to those with no comorbidities. ${ }^{82}$ Tenforde et al have confirmed that subjects with three or more co-morbidities (including psychiatric conditions and obesity) are associated with more than twofold odds of not returning to the basal health status. ${ }^{80}$
Another UK-based study on Long-COVID-19 subjects revealed that the underlying co-morbidities like asthma and obesity are associated with the Long-COVID-19 symptoms across all age groups. The association of cardiopulmonary diseases with the development of Long-COVID-19 was seen in elderly individuals aged more than 70 years. ${ }^{81}$

\section{Initial Symptoms of the Patients}

Few researchers have explored the predictors of LongCOVID-19 based on the symptoms seen during the early phase of the illness. Sudre et al have reported that individuals with more than five symptoms within the first week of presentation were at high risk for development of LongCOVID-19; and found it as the single, strongest predictive factor within gender and across all age groups. ${ }^{81}$ On further analysis of odds ratio (OR) for each individual; the five most common early symptoms experienced during the first week of acute illness in these patients were fatigue $(\mathrm{OR}=2.83)$, headache $(\mathrm{OR}=2.62)$, dyspnea $(\mathrm{OR}=2.36)$, 
hoarseness of voice $(\mathrm{OR}=2.33)$ and myalgia $(\mathrm{OR}=2.22) .{ }^{81}$ Additionally, it also revealed that the ongoing fever, loss of smell, multi-organ involvement, and underlying cardiopulmonary co-morbidity in the older age group should be taken as "red flag" signs. ${ }^{81}$

\section{Abnormal Laboratory Results}

The deranged blood indices (lymphopenia, neutrophillymphocyte ratio, thrombocytopenia, coagulation profile) and raised liver enzymes, inflammatory markers (eg, CRP, ferritin, IL-6), LDH, d-dimer, troponin, and creatine phosphokinase are associated with the worst outcomes in hospitalized subjects. ${ }^{83,84}$ Whether these abnormalities have a direct pathogenetic relationship with the occurrence of the Long-COVID-19 or whether these are just markers of a severely acute phase illness is not yet known. A prospective study by Spoto et al has revealed that mid-regional proAdrenomedullin (MR-proADM) is another important marker to predict disease severity and patients with its value $\geq 2 \mathrm{nmol} / 1$ are associated with higher mortality risk related to a multiorgan dysfunction syndrome. ${ }^{85}$ Some of the previous reports on lung injury (MERS-CoV related) have shown an increased risk of fibrosis in subjects with raised $\mathrm{LDH}^{86}$ There is evidence of persistent lymphopenia, high d-dimer values, and elevated $\mathrm{C}$ reactive protein (CRP) in $7.1 \%, 30.1 \%$, and $9.5 \%$ of Long-COVID-19 subjects. However, overall an improving trend of abnormal parameters was observed in LongCOVID-19 subjects compared to the initial acute phase laboratory derangement. ${ }^{45}$ In another study, more than $10 \%$ of the Long-COVID- 19 subjects showed high alanine transferase, lactate dehydrogenase, and mean corpuscular hemoglobin concentration. ${ }^{17}$

\section{Disease Severity, Length of ICU-Stay and Assisted Ventilation}

Though Long-COVID-19 symptoms are seen in subjects with mild acute illness, those with severe illness and requiring critical care show exaggerated predisposition for Long-COVID-19 illness. Often the severe and critically ill COVID-19 subjects require ICU admission and mechanical ventilation. The disease severity and associated co-morbid conditions determine the length of hospital stay and prolonged manifestations related to it. Previous studies have shown increasing evidence of pulmonary fibrosis and sequel secondary to ARDS. ${ }^{87}$ Prolonged critical illnesses are also associated with protracted symptoms and abnormalities, including fatigue, anemia, sarcopenia, and neuropsychiatric abnormalities. ${ }^{87}$ A recent study also reported the prolonged duration of hospital stay and higher intensive care admission frequency among the Long-COVID-19 subjects with evidence of fibrosis on CT chest imaging. ${ }^{88}$ This patient group also needed pulsed steroid therapy and antiviral therapy for a longer duration. ${ }^{87}$ In some instances, superadded fungal or bacterial pulmonary infection is also associated with significantly prolonged illness following acute COVID-19. ${ }^{89}$

\section{Management of Long-COVID-19}

Currently, the knowledge of Long-COVID-19 is sparse in most aspects, and there are no consensus guidelines for its management. Nevertheless, based on the availability of limited follow-up data, this vulnerable group of the COVID-19 recovered population requires a thorough clinical assessment to identify new, persistent, or progressive symptoms; and should be appropriately investigated. The Long-COVID-19 subjects need close follow-up for monitoring early, intermediate, and late complications. The requirement of oxygen supplementation, palliative care, rehabilitation, counseling, and other psycho-social needs should be assessed and addressed immediately. ${ }^{90}$ The patients should be advised and prepared for self-management and supported selfmanagement, and additional support is provided for older people and children with Long-COVID-19 symptoms. ${ }^{4}$ The more serious and potentially lifethreatening complications like pulmonary venous thromboembolism, stroke, and acute cardiac events must be identified early and appropriately managed; or referred to the care centers with subspecialty experts..$^{90}$ The utility of steroids, anticoagulants, and other medications is yet to be explored entirely, and the results of the longitudinal therapeutic clinical trials may address this issue in the future. However, special care must be taken while prescribing steroids to avoid secondary fungal infections like aspergillosis, mucormycosis and pneumocystis pneumonia especially in diabetic and immunocompromised patients. The role of pirfenidone and nintedanib is also being evaluated in reducing lung fibrosis and further pulmonary damage. ${ }^{91}$ For skin lesions, authors (based on their personal experience) suggest using topical steroids and oral anti-allergic drugs in mild to moderate cases and a short course of oral steroids in severe cases. A few authors have reported reduced exercise capacity in 
Long-COVID-19 subjects and suggested including aerobic exercises in rehabilitation of Long-COVID-19 subjects, which may boost immunity and respiratory functions. ${ }^{92}$ Besides, an advisory need to be given to all the patients for lifestyle modification and general preventive measures (like social distancing, hand hygiene, and the wearing of a face mask) to avoid reinfection. While managing the Long-COVID-19 subjects, care should be exercised to efficiently and optimally use the clinical, investigative, and therapeutic tools.

Multiple prospective or randomized trials are already initiated under the COVID follow-up facilities worldwide, and health scientists are calling for systematic collection of baseline and follow-up data of the Long-COVID-19 subjects. Multispecialty teams consisting of general physicians, radiologists, microbiologists, subspecialty experts, pathologists, and rehabilitative care specialists should be formed to deal with Long-COVID-19. The follow-up of Long-COVID -19 subjects should be kept flexible based on their ongoing clinical issues, rather than following any rigid pre-defined schedule. ${ }^{4}$ The policymakers and administrative personnel should establish the adequate infrastructure for follow-up and management of Long-COVID-19 and strengthen the existing facilities to treat COVID-19 illness.

\section{Conclusion}

With the re-emergence of the new waves of SARS-CoV-2 infection in many countries, it is expected that the burden of patients having long-term sequel of COVID-19 is going to be huge and it is expected to produce another public health crisis on the heels of current pandemic. Thus, it is extremely important to be aware of its protean clinical manifestations, risk predictors, and holistic management strategies. Further research comprising cataloging of symptoms, longer-ranging observational studies, and clinical trials are critical to evaluate the long-term consequences of COVID-19, and it warrants setting-up of dedicated, post-COVID care, multi-disciplinary clinics, and rehabilitation centers.

\section{Acknowledgment}

We thank Dr. Harish Bhujade (PGI, Chandigarh) for his valuable inputs and preparing the pictorial diagrams used in the manuscript.

\section{Disclosure}

The authors report no conflicts of interest in this work.

\section{References}

1. Garg S, Garg M, Prabhakar N, Malhotra P, Agarwal R. Unraveling the mystery of Covid-19 cytokine storm: from skin to organ systems. Dermatol Ther. 2020;33(6):1385-1389. doi:10.1111/dth.13859

2. Gupta A, Madhavan MV, Sehgal K, et al. Extrapulmonary manifestations of COVID-19. Nat Med. 2020;26:1017-1032. doi:10.1038/ s41591-020-0968-3

3. WHO [homepage on the internet]. Coronavirus disease (COVID-19). [updated November 10, 2020]. Available from: https://www.who.int/ news-room/q-a-detail/coronavirus-disease-covid-19/. Accessed December 14, 2020

4. Shah W, Hillman T, Playford ED, Hishmeh L. Managing the long term effects of covid-19: summary of NICE, SIGN, and RCGP rapid guideline. BMJ. 2021;372:n136. doi:10.1136/bmj.n136

5. Mahase E. Covid-19: what do we know about "long covid"? BMJ. 2020;370:m2815. doi:10.1136/bmj.m2815

6. Greenhalgh T, Knight M, A'Court C, Buxton M, Husain L. Management of post-acute covid-19 in primary care. BMJ. 2020;370:m3026. doi:10.1136/bmj.m3026

7. Callard F, Perego E. How and why patients made Long Covid. Soc Sci Med. 2021;268:113426. doi:10.1016/j.socscimed.2020.113426

8. Worldometer [Homepage on internet]. Coronavirus update. Available from: https://www.worldometers.info/coronavirus/. Accessed May 15,2021

9. Lopez-Leon S, Wegman-Ostrosky T, Perelman C, et al. More than 50 Long-term effects of COVID-19: a systematic review and meta-analysis. medRxiv [Preprint]. 2021. doi:10.1101/2021.01. 27.21250617

10. Barman MP, Rahman T, Bora K, Borgohain C. COVID-19 pandemic and its recovery time of patients in India: a pilot study. Diabetes Metab Syndr. 2020;14(5):1205-1211. doi:10.1016/j.dsx.2020.07.004

11. Nehme M, Braillard O, Alcoba G, et al. COVID-19 symptoms: longitudinal evolution and persistence in outpatient settings. Ann Intern Med. 2020.

12. Carfî A, Bernabei R, Landi F. Gemelli against COVID-19 post-acute care study group. Persistent symptoms in patients after acute COVID-19. JAMA. 2020;324:603-605. doi:10.1001/jama.2020. 12603

13. Office for National Statistics. The prevalence of long COVID symptoms and COVID-19 complications. [updated December 16, 2020]. Available from: https://www.ons.gov.uk/. Accessed February 2, 2021.

14. Goërtz YMJ, Van Herck M, Delbressine JM, et al. Persistent symptoms 3 months after a SARS-CoV-2 infection: the post-COVID-19 syndrome? ERJ Open Res. 2020;6:00542-2020. doi:10.1183/ 23120541.00542-2020

15. Garrigues E, Janvier P, Kherabi Y, et al. Post-discharge persistent symptoms and health-related quality of life after hospitalization for COVID-19. J Infect. 2020;81(6):e4-e6. doi:10.1016/j.jinf.2020. 08.029

16. Xiong Q, Xu M, Li J, et al. Clinical sequelae of COVID-19 survivors in Wuhan, China: a single-centre longitudinal study. Clin Microbiol Infect. 2021;27(1):89-95. doi:10.1016/j.cmi.2020.09.023

17. Dennis A, Wamil M, Alberts J; COVERSCAN study investigators. Multiorgan impairment in low-risk individuals with post-COVID-19 syndrome: a prospective, community-based study. BMJ Open. 2021;11(3):e048391. doi:10.1136/bmjopen-2020-048391

18. Carvalho-Schneider C, Laurent E, Lemaignen A, et al. Follow-up of adults with noncritical COVID-19 two months after symptom onset. Clin Microbiol Infect. 2021;27(2):258-263. doi:10.1016/j.cmi.20 20.09.052

19. Davis HE, Assaf GS, McCorkell L, et al. Characterizing Long COVID in an international cohort: 7 Months of symptoms and their impact. medRxiv [Preprint]. 2020. 
20. López Castro J. Post-COVID-19 syndrome (PC19S): chronic reactive endotheliitis and disseminated vascular disease. Acta Med Port. 2020;33(12):859. doi:10.20344/amp.14612

21. Afrin LB, Weinstock LB, Molderings GJ. Covid-19 hyperinflammation and post-Covid-19 illness may be rooted in mast cell activation syndrome. Int $J$ Infect Dis. 2020;100:327-332. doi:10.1016/j. ijid.2020.09.016

22. Sollini M, Ciccarelli M, Cecconi M, et al. Vasculitis changes in COVID-19 survivors with persistent symptoms: an $\left[{ }^{18} \mathrm{~F}\right] \mathrm{FDG}-\mathrm{PET} /$ CT study. Eur J Nucl Med Mol Imaging. 2020;30:1-7.

23. Aragão MFVV, Leal MC, Cartaxo Filho OQ, Fonseca TM, Valença MM. Anosmia in COVID-19 associated with injury to the olfactory bulbs evident on MRI. AJNR Am J Neuroradiol. 2020;41:1703-1706. doi:10.3174/ajnr.A6675

24. Strauss SB, Lantos JE, Heier LA, Shatzkes DR, Phillips CD. Olfactory bulb signal abnormality in patients with COVID-19 who present with neurologic symptoms. AJNR Am J Neuroradiol. 2020;41:1882-1887. doi:10.3174/ajnr.A6751

25. Roncati L, Ligabue G, Fabbiani L, et al. Type 3 hypersensitivity in COVID-19 vasculitis. Clin Immunol. 2020;217:108487. doi:10.1016/ j.clim.2020.108487

26. Liu Y, Sawalha AH, Lu Q. COVID-19 and autoimmune diseases. Curr Opin Rheumatol. 2021;33(2):155-162. doi:10.1097/ BOR.0000000000000776

27. Hussein HM, Rahal EA. The role of viral infections in the development of autoimmune diseases. Crit Rev Microbiol. 2019;45:394-412. doi:10.1080/1040841X.2019.1614904

28. Fujinami RS, von Herrath MG, Christen U, Whitton JL. Molecular mimicry, bystander activation, or viral persistence: infections and autoimmune disease. Clin Microbiol Rev. 2006;19:80-94. doi:10.1128/CMR.19.1.80-94.2006

29. Ehrenfeld M, Tincani A, Andreoli L, et al. Covid-19 and autoimmunity. Autoimmun Rev. 2020;19:102597. doi:10.1016/j. autrev.2020.102597

30. Zhou Y, Han T, Chen J, et al. Clinical and autoimmune characteristics of severe and critical cases of COVID-19. Clin Transl Sci. 2020;13:1077-1086. doi:10.1111/cts.12805

31. Herzog EL, Mathur A, Tager AM, et al. Interstitial lung disease associated with systemic sclerosis and idiopathic pulmonary fibrosis: how similar and distinct? Arthritis Rheumatol. 2014;66:1967-1978. doi:10.1002/art.38702

32. Zuo Y, Estes SK, Ali RA, et al. Prothrombotic autoantibodies in serum from patients hospitalized with COVID-19. Sci Transl Med. 2020;12(570):eabd3876. doi:10.1126/scitranslmed.abd3876

33. Zuo Y, Zuo M, Yalavarthi S, et al. Neutrophil extracellular traps and thrombosis in COVID-19. medRxiv. 2020.

34. Ye G, Pan Z, Pan Y, et al. Clinical characteristics of severe acute respiratory syndrome coronavirus 2 reactivation. J Infect. 2020;80 (5):14-17. doi:10.1016/j.jinf.2020.03.001

35. Investigative criteria for suspected cases of SARS-CoV-2 reinfection (ICR). https://www.cdc.gov/coronavirus/2019-ncov/php/investcriteria.html. Accessed February 3, 2021.

36. Long QX, Tang XJ, Shi QL, et al. Clinical and immunological assessment of asymptomatic SARS-CoV-2 infections. Nat Med. 2020;26(8):1200-1204. doi:10.1038/s41591020-0965-6

37. Wong AW, Shah AS, Johnston JC, Carlsten C, Ryerson CJ. Patientreported outcome measures after COVID-19: a prospective cohort study. Eur Respir J. 2020;56(5):2003276. doi:10.1183/139930 03.03276-2020

38. Chopra V, Flanders SA, O’Malley M, Malani AN, Prescott HC. Sixty-day outcomes among patients hospitalized with COVID-19. Ann Intern Med. 2020.

39. Garg M, Prabhakar N, Bhalla AS, et al. Computed tomography chest in COVID-19: when \& why? Indian J Med Res. 2021;153(12):86-92. doi:10.4103/ijmr.IJMR_3669_20
40. Garg M, Gupta P, Maralakunte M, et al. Diagnostic accuracy of CT and radiographic findings for novel coronavirus 2019 pneumonia: systematic review and meta-analysis. Clin Imaging. 2020;72:75-82. doi:10.1016/j.clinimag.2020.11.021

41. Wang Y, Dong C, Hu Y, et al. Temporal changes of CT findings in 90 patients with COVID-19 pneumonia: a longitudinal study. Radiology. 2020;296(2):E55-E64. doi:10.1148/radiol.2020200843

42. Udwadia ZF, Koul PA, Richeldi L. Post-COVID lung fibrosis: the tsunami that will follow the earthquake. Lung India. 2021;38:S41S47. doi:10.4103/lungindia.lungindia_818_20

43. Chun HJ, Coutavas E, Pine A, et al. Immuno-fibrotic drivers of impaired lung function in post-COVID-19 syndrome. medRxiv [Preprint]. 2021. doi:10.1101/2021.01.31.21250870

44. Mo X, Jian W, Su Z, et al. Abnormal pulmonary function in COVID-19 patients at time of hospital discharge. Eur Respir J. 2020;55(6):2001217. doi:10.1183/13993003.01217-2020

45. Mandal S, Barnett J, Brill SE, et al.; ARC Study Group. 'LongCOVID': a cross-sectional study of persisting symptoms, biomarker and imaging abnormalities following hospitalisation for COVID-19. Thorax. 2020. doi:10.1136/thoraxjnl-2020-215818

46. Inciardi RM, Lupi L, Zaccone G, et al. Cardiac involvement in a patient with coronavirus disease 2019 (COVID-19). JAMA Cardiol. 2020;5(7):819-824. doi:10.1001/jamacardio.2020.1096

47. Chen L, Li X, Chen M, Feng Y, Xiong C. The ACE2 expression in human heart indicates new potential mechanism of heart injury among patients infected with SARS-CoV-2. Cardiovasc Res. 2020;116:1097-1100. doi:10.1093/cvr/cvaa078

48. Varga Z, Flammer AJ, Steiger P, et al. Endothelial cell infection and endotheliitis in COVID-19. Lancet. 2020;395(10234):1417-1418. doi:10.1016/S0140-6736(20)30937-5

49. Xu Z, Shi L, Wang Y, et al. Pathological findings of COVID-19 associated with acute respiratory distress syndrome. Lancet Respir Med. 2020;8(4):420-422. doi:10.1016/S2213-2600(20)30076-X

50. Huang L, Zhao P, Tang D, et al. Cardiac involvement in patients recovered from COVID-2019 identified using magnetic resonance imaging. JACC Cardiovasc Imaging. 2020;13(11):2330-2339. doi:10.1016/j.jcmg.2020.05.004

51. Puntmann VO, Carerj ML, Wieters I, et al. Outcomes of cardiovascular magnetic resonance imaging in patients recently recovered from coronavirus disease 2019 (COVID-19). JAMA Cardiol. 2020;5 (11):1265-1273. doi:10.1001/jamacardio.2020.3557

52. Rajpal S, Tong MS, Borchers J, et al. Cardiovascular magnetic resonance findings in competitive athletes recovering from COVID-19 infection. JAMA Cardiol. 2021;6(1):116-118. doi:10. 1001/jamacardio.2020.4916

53. Dani M, Dirksen A, Taraborrelli P, et al. Autonomic dysfunction in 'long COVID': rationale, physiology and management strategies. Clin Med. 2021;21(1):e63-e67. doi:10.7861/clinmed.2020-0896

54. McMahon DE, Gallman AE, Hruza GJ, et al. Long COVID in the skin: a registry analysis of COVID-19 dermatological duration. Lancet Infect Dis. 2021;21(3):313-314. doi:10.1016/S1473-3099(20)30986-5

55. Mehta P, Bunker CB, Ciurtin C, et al. Chilblain-like acral lesions in long COVID-19: management and implications for understanding microangiopathy. Lancet Infect Dis. 2021. doi:10.1016/S14733099(21)00133-X

56. Suchonwanit P, Leerunyakul K, Kositkuljorn C. Cutaneous manifestations in COVID-19: lessons learned from current evidence. $J \mathrm{Am}$ Acad Dermatol. 2020;83:e57-e60. doi:10.1016/j.jaad.2020.04.094

57. Mao L, Jin H, Wang M, et al. Neurologic manifestations of hospitalized patients with coronavirus disease 2019 in Wuhan, China. JAMA Neurol. 2020;77(6):683-690. doi:10.1001/jamaneurol.2020.1127

58. Stefano GB, Ptacek R, Ptackova H, Martin A, Kream RM. Selective neuronal mitochondrial targeting in SARS-CoV-2 infection affects cognitive processes to induce 'Brain Fog' and results in behavioral changes that favor viral survival. Med Sci Monit. 2021;27:e930886. doi:10.12659/MSM.930886 
59. Lechien JR, Chiesa-Estomba CM, De Siati DR, et al. Olfactory and gustatory dysfunctions as a clinical presentation of mild-to-moderate forms of the coronavirus disease (COVID-19): a multicenter European study. Eur Arch Otorhinolaryngol. 2020;277 (8):2251-2261. doi:10.1007/s00405-020-05965-1

60. Lu Y, Li X, Geng D, et al. Cerebral micro-structural changes in COVID-19 patients - an MRI-based 3-month follow-up study. EClinicalMedicine. 2020;25:100484. doi:10.1016/j.eclinm.2020.10 0484

61. Helms J, Kremer S, Merdji H, et al. Neurologic features in severe SARS-CoV-2 infection. $N$ Engl $J$ Med. 2020;382(23):2268-2270. doi:10.1056/NEJMc2008597

62. Rogers JP, Chesney E, Oliver D, et al. Psychiatric and neuropsychiatric presentations associated with severe coronavirus infections: a systematic review and meta-analysis with comparison to the COVID-19 pandemic. Lancet Psychiatry. 2020;7(7):611-627. doi:10.1016/S2215-0366(20)30203-0

63. Parker AM, Sricharoenchai T, Raparla S, et al. Posttraumatic stress disorder in critical illness survivors: a metaanalysis. Crit Care Med. 2015;43:1121-1129. doi:10.1097/CCM.0000000000000882

64. Nikayin S, Rabiee A, Hashem MD, et al. Anxiety symptoms in survivors of critical illness: a systematic review and meta-analysis. Gen Hosp Psychiatry. 2016;43:23-29. doi:10.1016/j.genhosppsych. 2016.08.005

65. Rabiee A, Nikayin S, Hashem MD, et al. Depressive symptoms after critical illness: a systematic review and meta-analysis. Crit Care Med. 2016;44:1744-1753. doi:10.1097/CCM.00000000000 01811

66. Wohleb ES, Franklin T, Iwata M, Duman RS. Integrating neuroimmune systems in the neurobiology of depression. Nat Rev Neurosci. 2016;17:497-511. doi:10.1038/nrn.2016.69

67. Hopkins RO, Weaver LK, Pope D, et al. Neuropsychological sequelae and impaired health status in survivors of severe acute respiratory distress syndrome. Am J Respir Crit Care Med. 1999;160:50-56. doi:10.1164/ajrccm.160.1.9708059

68. Sansone A, Mollaioli D, Ciocca G, et al. Addressing male sexual and reproductive health in the wake of COVID-19 outbreak. $J$ Endocrinol Invest. 2021;44(2):223-231. doi:10.1007/s40618-020-01350-1

69. Anwar S, Acharya S, Shabih S, Khabut A. Acute limb ischemia in COVID-19 disease: a mysterious coagulopathy. Cureus. 2020;12(7): e9167.

70. Acharya S, Anwar S, Siddiqui FS, Shabih S, Manchandani U, Dalezman S. Renal artery thrombosis in COVID-19. IDCases. 2020;22:e00968. doi:10.1016/j.idcr.2020.e00968

71. Marinho PM, Marcos AAA, Romano AC, Nascimento H, Jr BR. Retinal findings in patients with COVID-19. Lancet. 2020;395 (10237):1610. doi:10.1016/S0140-6736(20)31014-X

72. Fernandez CE, Franz CK, Ko JH, et al. Imaging review of peripheral nerve injuries in patients with COVID-19. Radiology. 2021;298(3): E117-E130. doi:10.1148/radiol.2020203116

73. Wu Z, McGoogan JM. Characteristics of and important lessons from the coronavirus disease 2019 (COVID-19) outbreak in China: summary of a report of 72314 cases from the Chinese center for disease control and prevention. JAMA. 2020;323:1239-1242. doi:10.1001/ jama.2020.2648

74. Ojo AS, Balogun SA, Williams OT, Ojo OS. Pulmonary fibrosis in COVID-19 survivors: predictive factors and risk reduction strategies. Pulm Med. 2020;2020:6175964. doi:10.1155/2020/6175964

75. Sokolowska M, Lukasik ZM, Agache I, et al. Immunology of COVID-19: mechanisms, clinical outcome, diagnostics, and perspectives-a report of the European Academy of Allergy and Clinical Immunology (EAACI). Allergy. 2020;75:2445-2476. doi: $10.1111 /$ all.14462
76. Radzikowska U, Ding M, Tan G, et al. Distribution of ACE2, CD147, CD26, and other SARS-CoV-2 associated molecules in tissues and immune cells in health and in asthma, COPD, obesity, hypertension, and COVID-19 risk factors. Allergy. 2020;75:2829-2845. doi:10.11 11/all.14429

77. Dong X, Cao YY, Lu XX, et al. Eleven faces of coronavirus disease 2019. Allergy. 2020;75:1699-1709. doi:10.1111/all.14289

78. Verity R, Okell LC, Dorigatti I, et al. Estimates of the severity of coronavirus disease 2019: a model-based analysis. Lancet Infect Dis. 2020;20(6):669-677. doi:10.1016/S1473-3099(20)30243-7

79. Richardson S, Hirsch JS, Narasimhan M; the Northwell COVID-19 Research Consortium. Presenting characteristics, comorbidities, and outcomes among 5700 patients hospitalized with COVID-19 in the New York city area. JAMA. 2020;323(20):2052-2059. doi:10.1001/ jama.2020.6775

80. Tenforde MW, Kim SS, Lindsell CJ; CDC COVID-19 Response Team; IVY Network Investigators. Symptom duration and risk factors for delayed return to usual health among outpatients with COVID-19 in a multistate health care systems network - United States, March-June 2020. MMWR Morb Mortal Wkly Rep. 2020;69 (30):993-998. doi:10.15585/mmwr.mm6930e1

81. Sudre CH, Murray B, Varsaysky T, et al. Attributes and predictors of Long-COVID: analysis of COVID cases and their symptoms collected by the covid symptoms study app. medRxiv. 2020.

82. Stokes EK, Zambrano LD, Anderson KN, et al. Coronavirus disease 2019 case surveillance - United States, January 22-May 30, 2020. MMWR Morb Mortal Wkly Rep. 2020;69(24):759-765. doi:10.15585/ mmwr.mm6924e2

83. Liao D, Zhou F, Luo L, et al. Haematological characteristics and risk factors in the classification and prognosis evaluation of COVID-19: a retrospective cohort study. Lancet Haematol. 2020;7(9):e671-e678. doi:10.1016/S2352-3026(20)30217-9

84. Del Valle DM, Kim-Schulze S, Huang HH, et al. An inflammatory cytokine signature predicts COVID-19 severity and survival. Nat Med. 2020;26(10):1636-1643. doi:10.1038/s41591-020-1051-9

85. Spoto S, Agrò FE, Sambuco F, et al. High value of mid-regional proadrenomedullin in COVID-19: a marker of widespread endothelial damage, disease severity, and mortality. J Med Virol. 2021;93 (5):2820-2827. doi:10.1002/jmv.26676

86. Das KM, Lee EY, Singh R, et al. Follow-up chest radiographic findings in patients with MERS-CoV after recovery. Indian J Radiol Imaging. 2017;27(3):342-349. doi:10.4103/ijri.IJRI_469_16

87. Oeckler RA, Hubmayr RD. Ventilator-associated lung injury: a search for better therapeutic targets. Eur Respir J. 2007;30:1216-1226. doi:10.1183/09031936.00104907

88. Yu M, Liu Y, Xu D, et al. Prediction of the development of pulmonary fibrosis using serial thin-section $\mathrm{CT}$ and Clinical features in patients discharged after treatment for COVID-19 pneumonia. Korean J Radiol. 2020;21(6):746-755. doi:10.3348/kjr.2020.0215

89. Zhou P, Liu Z, Chen Y, et al. Bacterial and fungal infections in COVID-19 patients: a matter of concern. Infect Control Hosp Epidemiol. 2020;41(9):1124-1125. doi:10.1017/ice.2020.156

90. George PM, Barratt SL, Condliffe R, et al. Respiratory follow-up of patients with COVID-19 pneumonia. Thorax. 2020;75 (11):1009-1016. doi:10.1136/thoraxjnl-2020-215314

91. George PM, Wells AU, Jenkins RG. Pulmonary fibrosis and COVID-19: the potential role for antifibrotic therapy. Lancet Respir Med. 2020;8:807-815. doi:10.1016/S2213-2600(20)30225-3

92. Mohamed AA, Alawna M. Role of increasing the aerobic capacity on improving the function of immune and respiratory systems in patients with coronavirus (COVID-19): a review. Diabetes Metab Syndr. 2020;14(4):489-496. doi:10.1016/j.dsx.2020.04.038 


\section{Publish your work in this journal}

The International Journal of General Medicine is an international, peer-reviewed open-access journal that focuses on general and internal medicine, pathogenesis, epidemiology, diagnosis, monitoring and treatment protocols. The journal is characterized by the rapid reporting of reviews, original research and clinical studies across all disease areas. The manuscript management system is completely online and includes a very quick and fair peer-review system, which is all easy to use. Visit http://www.dovepress.com/ testimonials.php to read real quotes from published authors.

Submit your manuscript here: https://www.dovepress.com/international-journal-of-general-medicine-journal 